\title{
Stereoselective total synthesis of sphingolipids
}

\author{
PARAMESH JANGILI, PERLA RAMESH and BISWANATH DAS* \\ Natural Products Chemistry Division, Indian Institute of Chemical Technology, Tarnaka, Hyderabad, \\ Telangana 500 007, India \\ e-mail: biswanathdas@yahoo.com
}

MS received 13 July 2016; revised 9 September 2016; accepted 9 September 2016

\begin{abstract}
A novel sphingosine, 1,2-diacetyl D-erythro-sphinganine having a characteristic almond flavour was isolated from the edible mushroom Grifola gargal. We have synthesized this sphinganine along with the three other sphingolipids, such as 1,2-diacetyl L-threo-sphinganine, D-erythro-sphinganine triacetate and L-threo-sphinganine triacetate using Garner aldehyde as the starting material involving the Grignard reaction and Mitsunobu inversion. The sphingolipids 1,2-diacetyl D-erythro-sphinganine and 1,2-diacetyl L-threo-sphinganine have been synthesized for the first time.
\end{abstract}

Keywords. 1,2-Diacetyl D-erythro-sphinganine; 1,2-diacetyl L-threo-sphinganine; D-erythro-sphinganine triacetate; sphingolipids; total synthesis; Garner aldehyde.

\section{Introduction}

Sphingolipids are important structural and functional components of essentially all eukaryotic cells and are abundantly located in all plasma membranes as well as in some intracellular organelles. ${ }^{1,2}$ They exist as structural components of cell membranes in animals, plants and some microbial systems. ${ }^{3}$ Sphingosine $\mathbf{1}$ and sphinganine (dihydrosphingosine) $\mathbf{2}$ (Figure 1) are naturally occurring bioactive compounds (long-chain, aliphatic, 2-amino-1,3 diols). Dihydrosphingosines are biosynthetic precursors of sphingolipids (e.g., ceramides, sphingomyelin, cerebrosides and gangliosides), which play important roles in biological pathways such as cell regulation and signal transduction. Sphingoid bases contain two chiral centres, viz., at carbon atoms 2 and 3. Natural sphingoid bases occur in the D-erythro $(2 S, 3 R)$ configuration, but other additional unnatural isomers have also been reported. Among the unnatural sphingoid bases L-threo- $(2 S, 3 S)$ dihydrosphingosine (safingol) 3 (Figure 1) is of particular interest due to its medicinal importance. Safingol is an antineoplastic, antipsoriatic drug ${ }^{4}$ and a competitive inhibitor of protein kinase C. ${ }^{5}$

Recently, Choi et al., isolated ${ }^{6}$ a novel sphingosine, 1,2-diacetyl D-erythro-sphinganine (4) (Figure 2), from the edible mushroom Grifola gargal, having a characteristic almond flavour. This mushroom is collected

\footnotetext{
*For correspondence

Part 86 in the series "Synthetic studies on natural products"
}

and eaten by native people of southern Argentina and Chile. The compound $\mathbf{4}$ suppresses the formation of osteoclasts.

In continuation of our work carried out on the synthesis of natural bioactive compounds, ${ }^{7}$ herein we describe an efficient stereoselective total synthesis of naturally occurring sphingolipid 1,2-diacetyl Derythro-sphinganine (4) along with three other sphingolipids, such as 1,2-diacetyl L-threo-sphinganine (5) (C-3 epimer of 4), D-erythro-sphinganine triacetate (6) (triacetyl derivative of compound 2) and L-threosphinganine triacetate (7) (triacetyl derivative of compound 3, safingol) (Figure 2). Our planned approach to the synthesis of the target molecules was initiated from Garner aldehyde (9) involving the Grignard reaction and Mitsunobu inversion. To our knowledge, there are a few reports towards the total synthesis of sphingolipids $\mathbf{6}$ and 7. ${ }^{8}$ However, synthesis of the sphingolipids $\mathbf{4}$ and 5 are reported here for the first time.

\section{Experimental}

\subsection{General}

Infrared spectra were recorded on Perkin-Elmer RX1 FT-IR spectrophotometer. NMR spectra were recorded on Inova $500 \mathrm{MHz}$ and Bruker $300 \mathrm{MHz}$ spectrometers using $\mathrm{CDCl}_{3}$ and $\mathrm{CD}_{3} \mathrm{OD}$ as solvents and $\mathrm{Me}_{4} \mathrm{Si}$ as internal standard. The chemical shifts are expressed as $\delta$ values in parts per million (ppm) and the coupling constants $(J)$ are given in hertz $(\mathrm{Hz})$. ESIMS were recorded with VG-Autospec micromass. Optical rotations were 


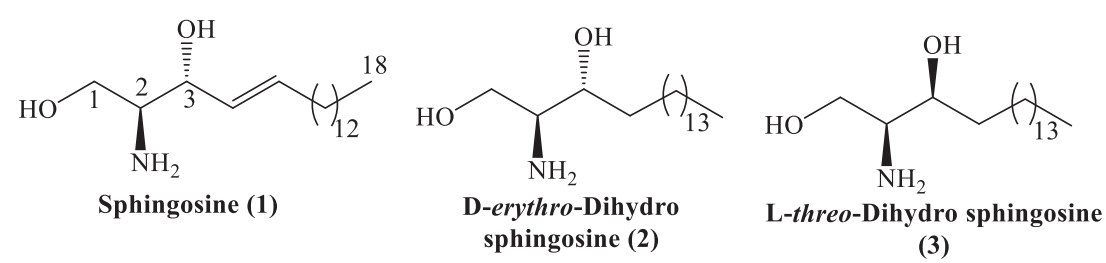

Figure 1. Structures of sphingosine (1) and sphinganines (dihydro sphingosines) (2 and 3).
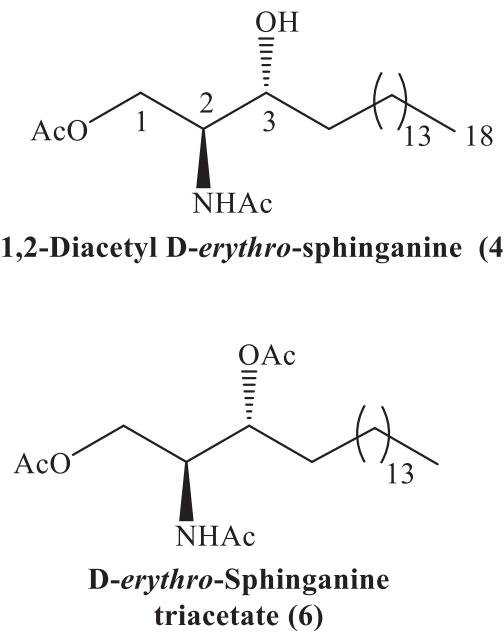<smiles>CCC[C@H](O)[C@@H](N)COC(C)=O</smiles>

1,2-Diacetyl L-threo-sphinganine (5)

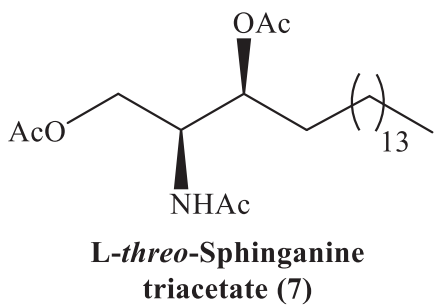

Figure 2. Structures of synthesized sphinganines (4-7).

measured with JASCO DIP 360 digit polarimeter. All reactions were monitored by thin-layer chromatography (TLC) using silica gel $F_{254}$ pre-coated plates.

\section{2 tert-Butyl (S)-4-((S)-1-hydroxyhexadecyl)-2, 2-dimethyloxa zolidine-3-carboxylate (10)}

To the solution of Garner aldehyde (9) $(2.0 \mathrm{~g}, 8.72 \mathrm{mmol})$ in THF $(10 \mathrm{~mL})$, was added at $-78{ }^{\circ} \mathrm{C}$ pentadecyl magnesium bromide in THF $(10 \mathrm{~mL})$ which was prepared from 1-bromopentadecane $(7.58 \mathrm{~mL}, 26.16 \mathrm{mmol})$ and $\mathrm{Mg}(0.847 \mathrm{~g}, 34.88 \mathrm{mmol})$ in the usual manner. The mixture was stirred at the same temperature $\left(-78{ }^{\circ} \mathrm{C}\right)$ for 1 hour and then gradually brought to r.t. The mixture was then stirred overnight at r.t. to produce the mixture of diastereomers $\mathbf{1 0}$ and $\mathbf{1 0 a}$. The reaction was quenched by the addition of aqueous saturated $\mathrm{NH}_{4} \mathrm{Cl}$ $(10 \mathrm{~mL})$ and extracted with AcOEt. The organic layer was washed with $5 \%$ aqueous $\mathrm{HCl}(10 \mathrm{~mL})$, water, brine and then dried over $\mathrm{Na}_{2} \mathrm{SO}_{4}$. The two diastereomers 10 and 10a were separated by careful CC (silica gel, 100-200 mesh, 0-5\% increasing amount of AcOEt in hexane) to produce two diastereomeric alcohols 10 and 10a in the ratio 9:1 (syn:anti, 9:1) with $86 \%$ yield. The pure alcohol 10 (2.98 g) was obtained as a colorless oil. $[\alpha]_{\mathrm{D}}^{25}=-32.2\left(c=2.0, \mathrm{CHCl}_{3}\right)$. IR $\tilde{\mathrm{o}}_{\max }(\mathrm{KBr}) / \mathrm{cm}^{-1}: 3440,2925,2855,1701,1366,1258$, 1175, 1061. ${ }^{1} \mathrm{H}$ NMR $\left(500 \mathrm{MHz}, \mathrm{CDCl}_{3}\right): 4.10-3.49$ $(m, 4 \mathrm{H}) ; 1.62-1.55(m, 2 \mathrm{H}) ; 1.49(s, 12 \mathrm{H}) ; 1.45(s, 3 \mathrm{H})$; 1.25 (br. $s, 26 \mathrm{H}) ; 0.88(t, J=6.8 \mathrm{~Hz}, 3 \mathrm{H}) .{ }^{13} \mathrm{C} \mathrm{NMR}$ $\left(125 \mathrm{MHz}, \mathrm{CDCl}_{3}\right): 154.1 ; 94.2 ; 81.0 ; 72.9 ; 64.7 ; 62.3$; $34.4 ; 32.7 ; 31.8 ; 29.6 ; 29.6 ; 29.5 ; 28.3 ; 26.4 ; 26.0 ; 24.2$; 22.6; 14.0. ESIMS: $m / z 442[\mathrm{M}+\mathrm{H}]^{+}$. Anal Calcd. for $\mathrm{C}_{26} \mathrm{H}_{51} \mathrm{NO}_{4}$ : C, 70.70; H, 11.64\%. Found: C, 70.60; H, $11.68 \%$.

\section{3 tert-Butyl ((2S,3S)-1,3-dihydroxyoctadecan-2-yl) carbamate (11)}

Compound 10 (2.8 g, $6.34 \mathrm{mmol}$ ) and pyridinium $p$ toluene sulfonate $(0.159 \mathrm{~g}, 0.634 \mathrm{mmol})$ were dissolved in $\mathrm{MeOH}(10 \mathrm{~mL})$ and stirred at r.t. for $2 \mathrm{~h}$. The solvent was removed under reduced pressure. The residue was purified by flash CC (silica gel, hexane/AcOEt, 7:3) to give pure compound $\mathbf{1 1}(2.34 \mathrm{~g}, 92 \%)$ as a white solid. $[\alpha]_{\mathrm{D}}^{25}=+16.2\left(c=2.5, \mathrm{CHCl}_{3}\right) . \mathrm{IR} \tilde{\mathrm{o}}_{\max }(\mathrm{KBr}) / \mathrm{cm}^{-1}$ : $3430,2975,2855,1690,1360,1255,1175,1060 .{ }^{1} \mathrm{H}$ $\operatorname{NMR}\left(500 \mathrm{MHz}, \mathrm{CDCl}_{3}\right): 5.22(d, J=7.9 \mathrm{~Hz}, 1 \mathrm{H})$; $4.01(d d, J=3.4,11.4 \mathrm{~Hz}, 1 \mathrm{H}) ; 3.82(d, J=3.8 \mathrm{~Hz}$, $1 \mathrm{H}) ; 3.76(\mathrm{~m}, 1 \mathrm{H}) ; 3.53(\mathrm{~m}, 1 \mathrm{H}) ; 2.54$ (br. $s, 2 \mathrm{H})$; 1.56$1.48(m, 2 \mathrm{H}) ; 1.46(s, 9 \mathrm{H}) ; 1.25$ (br. $s, 26 \mathrm{H}) ; 0.88$ ( $t$, $J=6.7 \mathrm{~Hz}, 3 \mathrm{H}) .{ }^{13} \mathrm{C} \mathrm{NMR}\left(125 \mathrm{MHz}, \mathrm{CDCl}_{3}\right): 156.1$; $79.6 ; 73.7 ; 62.3 ; 54.9 ; 34.2 ; 31.8 ; 29.6 ; 29.5 ; 29.3$; $28.3 ; 25.9 ; 22.6 ; 14.0$. ESIMS: $m / z 402[\mathrm{M}+\mathrm{H}]^{+}$. Anal Calcd. for $\mathrm{C}_{23} \mathrm{H}_{47} \mathrm{NO}_{4}$ : C, 68.78; $\mathrm{H}, 11.80 \%$. Found: $\mathrm{C}$, $68.90 ; \mathrm{H}, 11.75 \%$. 


\section{4 (2S,3S)-2-((tert-Butoxycarbonyl)amino)-3-} hydroxy octadecyl acetate (8)

To a solution of 1,3-diol $11(2.2 \mathrm{~g}, 5.47 \mathrm{mmol})$ in dry $\mathrm{CH}_{2} \mathrm{Cl}_{2}(10 \mathrm{~mL}), \mathrm{Et}_{3} \mathrm{~N}(0.76 \mathrm{~mL}, 5.47 \mathrm{mmol})$ was added, followed by $\mathrm{Ac}_{2} \mathrm{O}(0.51 \mathrm{~mL}, 5.47 \mathrm{mmol})$ was added at $0^{\circ} \mathrm{C}$ and the reaction mixture was allowed to r.t. After completion of reaction $(2 \mathrm{~h})$, the reaction mixture was washed with brine $(10 \mathrm{~mL})$ and water $(10 \mathrm{~mL})$, dried over $\mathrm{Na}_{2} \mathrm{SO}_{4}$ and evaporated. The crude product was purified by silica gel CC (hexane/AcOEt, 9:1) to give pure monoacetyl ester $8(1.87 \mathrm{~g}, 77 \%)$ as a white solid. $[\alpha]_{\mathrm{D}}^{25}=+3.4\left(c=0.6, \mathrm{CHCl}_{3}\right)$. IR $\widetilde{\mathrm{O}}_{\max }(\mathrm{KBr}) / \mathrm{cm}^{-1}: 3350,2917,2850,1743,1685,1531$, 1367, 1232, 1173, 1049. ${ }^{1} \mathrm{H}$ NMR $\left(300 \mathrm{MHz}, \mathrm{CDCl}_{3}\right)$ : $4.91(d, J=8.0 \mathrm{~Hz}, 1 \mathrm{H}) ; 4.28-4.17(m, 1 \mathrm{H}) ; 4.06$ $(d d, J=6.1,11.0 \mathrm{~Hz}, 1 \mathrm{H}) ; 3.79(m, 1 \mathrm{H}) ; 3.69-3.58$ (m, 1H); 2.32 (br. $s, 1 \mathrm{H}) ; 2.08(s, 3 \mathrm{H}) ; 1.55-1.48$ $(m, 2 \mathrm{H}) ; 1.45(s, 9 \mathrm{H}) ; 1.25(s, 26 \mathrm{H}) ; 0.88(t, J=7.0$ $\mathrm{Hz}, 3 \mathrm{H}) .{ }^{13} \mathrm{C}$ NMR $\left(75 \mathrm{MHz}, \mathrm{CDCl}_{3}\right): 171.2 ; 156.0$; $79.6 ; 70.1 ; 63.4 ; 52.7 ; 33.7 ; 31.9 ; 29.7 ; 29.6 ; 29.5$; $29.3 ; 28.3 ; 25.6 ; 22.7 ; 20.9 ; 14.1$. ESI MS: $m / z 466$ $[\mathrm{M}+\mathrm{Na}]^{+}$. Anal Calcd. for $\mathrm{C}_{25} \mathrm{H}_{49} \mathrm{NO}_{5}: \mathrm{C}, 67.68 ; \mathrm{H}$, $11.13 \%$. Found: C, 67.83; H, 11.09\%.

\subsection{1,2-Diacetyl L-threo-sphinganine (5)}

To a solution of compound $8(0.3 \mathrm{~g}, 0.68 \mathrm{mmol})$ in $\mathrm{CH}_{2} \mathrm{Cl}_{2}(5 \mathrm{~mL})$ excess trifluoroacetic acid (TFA) was added dropwise and stirred at r.t. for $1 \mathrm{~h}$. The reaction mixture was dried on the rotary evaporator to remove the excess TFA. The resulting residue (unprotected amine) was dissolved in $\mathrm{CH}_{2} \mathrm{Cl}_{2}(5 \mathrm{~mL})$ and basified to $\mathrm{pH} 8$ with aq. $\mathrm{NaHCO}_{3}$ followed by the addition of the acetyl chloride $(0.053 \mathrm{~mL}, 0.75 \mathrm{mmol})$. The reaction was monitored by TLC. Upon completion $(2.5 \mathrm{~h})$, the reaction mixture was diluted with saturated aqueous $\mathrm{NH}_{4} \mathrm{Cl}$. The phases were separated and the aqueous layer was extracted with $\mathrm{CH}_{2} \mathrm{Cl}_{2}$. The combined organic extracts were washed with brine, dried $\left(\mathrm{Na}_{2} \mathrm{SO}_{4}\right)$ and purified by $\mathrm{CC}$ to afford pure compound $5(0.187 \mathrm{~g}, 72 \%)$ as a white solid. [á] $]_{\mathrm{D}}^{24}=+2.8$ $\left(c=0.4, \mathrm{CHCl}_{3}\right)$. IR $\tilde{\mathrm{o}}_{\max }(\mathrm{KBr}) / \mathrm{cm}^{-1}: 3300,2945$, 2840, 1725, 1645, 1540, 1435, 1365, 1061. ${ }^{1} \mathrm{H}$ NMR $\left(500 \mathrm{MHz}, \mathrm{CDCl}_{3}\right): 4.24(d d, J=4.0,10.0 \mathrm{~Hz}$, $1 \mathrm{H})$; 4.18-4.02 (m, 2H); $3.68(m, 1 \mathrm{H}) ; 2.62$ (br. $s$, $1 \mathrm{H}) ; 2.09(s, 3 \mathrm{H}) ; 2.02(s, 3 \mathrm{H}) ; 1.45(m, 1 \mathrm{H}) ; 1.25$ $(s, 27 \mathrm{H}) ; 0.88(t, J=6.9 \mathrm{~Hz}, 3 \mathrm{H}) .{ }^{13} \mathrm{C}$ NMR $(125$ $\left.\mathrm{MHz}, \mathrm{CDCl}_{3}\right): 171.6 ; 170.4 ; 69.7 ; 63.4 ; 51.5 ; 33.7$; $31.9 ; 29.6 ; 29.5 ; 29.5 ; 29.3 ; 25.7 ; 23.3 ; 22.7 ; 20.9$; 14.1. ESI MS: $m / z 386[\mathrm{M}+\mathrm{H}]^{+}$. Anal Calcd. for $\mathrm{C}_{22} \mathrm{H}_{43} \mathrm{NO}_{4}$ : C, 68.53; H, 11.24\%. Found: C, 68.62; $\mathrm{H}, 11.21 \%$.

\subsection{L-threo-Sphinganine triacetate (7)}

To a solution of compound $5(0.1 \mathrm{~g}, 0.26 \mathrm{mmol})$ in dry $\mathrm{CH}_{2} \mathrm{Cl}_{2}(5 \mathrm{~mL}), \mathrm{Et}_{3} \mathrm{~N}(0.043 \mathrm{~mL}, 0.31 \mathrm{mmol})$ was added, followed by $\mathrm{Ac}_{2} \mathrm{O}(0.029 \mathrm{~mL}, 0.31 \mathrm{mmol})$ was added at $0^{\circ} \mathrm{C}$ and the reaction mixture was allowed to rise to r.t. After $2 \mathrm{~h}$, the reaction mixture was washed with brine $(5 \mathrm{~mL})$ and water $(5 \mathrm{~mL})$, dried over $\mathrm{Na}_{2} \mathrm{SO}_{4}$ and evaporated. The crude product was purified by silica gel CC (hexane/AcOEt, 9:1) to give L-threosphinganine triacetate $7(0.087 \mathrm{~g}, 79 \%)$ as a white solid. $[\mathrm{a}]_{\mathrm{D}}^{24}=-12.0\left(c=0.5, \mathrm{CHCl}_{3}\right)$. IR $\tilde{\mathrm{o}}_{\max }(\mathrm{KBr}) / \mathrm{cm}^{-1}$ : 2925, 2855, 1740, 1655, 1543, 1460, 1370, 1235, 1048. ${ }^{1} \mathrm{H}$ NMR $\left(500 \mathrm{MHz}, \mathrm{CDCl}_{3}\right): 5.64(d, J=9.3 \mathrm{~Hz}, 1 \mathrm{H})$; $5.06(m, 1 \mathrm{H}) ; 4.39(m, 1 \mathrm{H}) ; 4.07-4.01(m, 2 \mathrm{H}) ; 2.05$ $(s, 3 \mathrm{H}) ; 2.02(s, 3 \mathrm{H}) ; 1.99(s, 3 \mathrm{H}) ; 1.62-1.54(m, 2 \mathrm{H})$; 1.24 (br. $s, 26 \mathrm{H}) ; 0.87(t, J=6.7 \mathrm{~Hz}, 3 \mathrm{H}) .{ }^{13} \mathrm{C} \mathrm{NMR}$ $\left(125 \mathrm{MHz}, \mathrm{CDCl}_{3}\right): 170.7 ; 170.4 ; 170.0 ; 72.4 ; 63.3$; $50.0 ; 31.9 ; 31.2 ; 29.6 ; 29.6 ; 29.5 ; 29.3 ; 29.2 ; 25.1 ; 23.2$; $22.6 ; 20.9 ; 20.7 ; 14.1$. ESI MS: $m / z 428[\mathrm{M}+\mathrm{H}]^{+}$. Anal Calcd. for $\mathrm{C}_{24} \mathrm{H}_{45} \mathrm{NO}_{5}$ : C, $67.41 ; \mathrm{H}, 10.61 \%$. Found: $\mathrm{C}$, $67.25 ; \mathrm{H}, 10.57 \%$.

\section{7 (2S,3R)-2-((tert-Butoxycarbonyl)amino) octadecane-1,3-diyl diacetate (12)}

The monoacetyl ester 8 (1.0 g, $2.25 \mathrm{mmol})$ was dissolved in anhydrous THF $(20 \mathrm{~mL}), \mathrm{AcOH}(0.257 \mathrm{~mL}$, $4.50 \mathrm{mmol})$ and $\mathrm{PPh}_{3}$ (1.18 g, $\left.4.50 \mathrm{mmol}\right)$ were added at $0{ }^{\circ} \mathrm{C}$. To the reaction mixture, a solution of diisopropyl azodicarboxylate $(0.88 \mathrm{~mL}, 4.50 \mathrm{mmol})$ in anhydrous THF $(10 \mathrm{~mL})$ was added. The solution was stirred at r.t. and after $2 \mathrm{~h}$ the reaction was quenched by the addition of water $(10 \mathrm{~mL})$, and extracted with $\mathrm{Et}_{2} \mathrm{O}(30$ $\mathrm{mL}$ ). After phase separation the organic phase was dried $\left(\mathrm{Na}_{2} \mathrm{SO}_{4}\right)$, concentrated and purified by $\mathrm{CC}$ to give pure $12(0.843 \mathrm{~g}, 77 \%)$ as a colorless oil. $[\alpha]_{\mathrm{D}}^{25}=+12.1$ $\left(c=0.3, \mathrm{CHCl}_{3}\right)$. IR $\tilde{\mathrm{o}}_{\max }(\mathrm{KBr}) / \mathrm{cm}^{-1}: 2925,2854$, 1746, 1723, 1368, 1236, 1171. ${ }^{1} \mathrm{H}$ NMR $(300 \mathrm{MHz}$, $\left.\mathrm{CDCl}_{3}\right)$ : $5.02(m, 1 \mathrm{H}) ; 4.92(d d, J=6.2,12.5 \mathrm{~Hz}, 1 \mathrm{H})$; 4.09-3.99 ( $m, 2 \mathrm{H})$; $2.06(s, 6 \mathrm{H})$; 1.67-1.57 ( $m, 2 \mathrm{H})$; $1.45(s, 9 \mathrm{H}) ; 1.25$ (br. $s, 26 \mathrm{H}) ; 0.88(t, J=6.8 \mathrm{~Hz}$, $3 \mathrm{H}) .{ }^{13} \mathrm{C}$ NMR $\left(75 \mathrm{MHz}, \mathrm{CDCl}_{3}\right): 170.8 ; 168.6 ; 151.3$; $79.9 ; 72.3 ; 62.9 ; 51.2 ; 31.9 ; 31.2 ; 29.6 ; 29.6 ; 29.5 ; 29.4$; $29.3 ; 28.3 ; 25.0 ; 22.6 ; 21.0 ; 20.8 ; 14.1$. ESI MS: $m / z$ $486[\mathrm{M}+\mathrm{H}]^{+}$. Anal Calcd. for $\mathrm{C}_{27} \mathrm{H}_{51} \mathrm{NO}_{6}: \mathrm{C}, 66.77 ; \mathrm{H}$, $10.58 \%$. Found: C, $66.85 ; \mathrm{H}, 10.54 \%$.

\subsection{D-erythro-Sphinganine triacetate (6)}

To a solution of compound $12(0.8 \mathrm{~g}, 1.64 \mathrm{mmol})$ in $\mathrm{CH}_{2} \mathrm{Cl}_{2}(5 \mathrm{~mL})$, excess trifluoroacetic acid was added dropwise and stirred at r.t. for $1 \mathrm{~h}$. The reaction mixture 
was dried on the rotary evaporator to remove the excess TFA. The resulting residue (unprotected amine) was dissolved in $\mathrm{CH}_{2} \mathrm{Cl}_{2}(8 \mathrm{~mL})$ and basified to $\mathrm{pH} 8$ with aq. $\mathrm{NaHCO}_{3}$ followed by the addition of the acetyl chloride $(0.128 \mathrm{~mL}, 1.80 \mathrm{mmol})$. The reaction was monitored by TLC. Upon completion $(2.5 \mathrm{~h})$, the reaction mixture was diluted with saturated aqueous $\mathrm{NH}_{4} \mathrm{Cl}$. The phases were separated and the aqueous layer was extracted with $\mathrm{CH}_{2} \mathrm{Cl}_{2}$. The combined organic extracts were washed with brine, dried $\left(\mathrm{Na}_{2} \mathrm{SO}_{4}\right)$ and purified by $\mathrm{CC}$ to afford pure $\mathrm{D}$-erythro-sphinganine triacetate $6(0.507 \mathrm{~g}, 72 \%)$ as a white solid. [á $]_{\mathrm{D}}^{24}=+12.7(c=$ $\left.1.1, \mathrm{CHCl}_{3}\right)$. IR $\tilde{\mathrm{o}}_{\max }(\mathrm{KBr}) / \mathrm{cm}^{-1}: 2935,2840,1729$, 1646, 1540, 1462, 1360, 1239, 1061. ${ }^{1} \mathrm{H}$ NMR (500 $\left.\mathrm{MHz}, \mathrm{CDCl}_{3}\right): 5.90(d, J=9.0 \mathrm{~Hz}, 1 \mathrm{H}) ; 4.90(m$, $1 \mathrm{H}) ; 4.38(m, 1 \mathrm{H}) ; 4.25(d d, J=6.1,11.5 \mathrm{~Hz}, 1 \mathrm{H})$; $4.06(d, J=4.0,11.7 \mathrm{~Hz}, 1 \mathrm{H}) ; 2.07(s, 3 \mathrm{H}) ; 2.06(s$, $3 \mathrm{H},) ; 2.00(s, 3 \mathrm{H}) ; 1.60(m, 2 \mathrm{H}) ; 1.24$ (br. $s, 26 \mathrm{H}) ;$, $(t, J=7.0 \mathrm{~Hz}, 3 \mathrm{H}) .{ }^{13} \mathrm{C} \mathrm{NMR}\left(125 \mathrm{MHz}, \mathrm{CDCl}_{3}\right)$ : $170.9 ; 170.9 ; 169.7 ; 74.0 ; 62.5 ; 50.5 ; 31.9 ; 31.4 ; 29.6$; $29.6 ; 29.5 ; 29.4 ; 29.3 ; 25.3 ; 22.6 ; 20.9 ; 20.8 ; 14.1$. ESI MS: $m / z 428[\mathrm{M}+\mathrm{H}]^{+}$. Anal Calcd. for $\mathrm{C}_{24} \mathrm{H}_{45} \mathrm{NO}_{5}$ : $\mathrm{C}$, $67.41 ; \mathrm{H}, 10.61 \%$. Found: C, 67.30; H, 10.64\%.

\section{$2.9 N-((2 S, 3 R)-1,3-D i h y d r o x y o c t a d e c a n-2-y l)$ acetamide (13)}

To a solution of triacetate $6(0.4 \mathrm{~g}, 0.935 \mathrm{mmol})$ in absolute $\mathrm{MeOH}(6 \mathrm{~mL})$ was added anhydrous $\mathrm{Na}_{2} \mathrm{CO}_{3}$ $(0.118 \mathrm{~g}, 1.12 \mathrm{mmol})$. The mixture was stirred at r.t. for $1 \mathrm{~h}$. Then, the solution was filtered, the solvent evaporated and the solid residue was purified over silica gel (eluent, $\mathrm{CH}_{2} \mathrm{Cl}_{2}$ with increasing amount of $\mathrm{MeOH}$ ) to give 1,3-diol $13(0.263 \mathrm{~g}, 82 \%)$ as a white solid. $[\alpha]_{\mathrm{D}}^{25}=$ $+6.6\left(c=0.2, \mathrm{CH}_{3} \mathrm{OH}\right)$. IR $\tilde{\mathrm{o}}_{\max }(\mathrm{KBr}) / \mathrm{cm}^{-1}: 3400$, 2940, 2835, 1650, 1548, 1430, 1362, 1061. ${ }^{1} \mathrm{H}$ NMR (500 MHz, $\left.\mathrm{CD}_{3} \mathrm{OD}\right): 3.86(m, 1 \mathrm{H}) ; 3.72-3.64(m, 2 \mathrm{H})$; $3.59(m, 1 \mathrm{H}) ; 1.97(s, 3 \mathrm{H}) ; 1.52(m, 2 \mathrm{H}) ; 1.28$ (br. $s$, $26 \mathrm{H}) ; 0.89(t, 3 \mathrm{H}, J=7.0 \mathrm{~Hz}) .{ }^{13} \mathrm{C}$ NMR $(125 \mathrm{MHz}$, $\left.\mathrm{CD}_{3} \mathrm{OD}\right): 173.4 ; 72.3 ; 62.1 ; 57.0 ; 34.8 ; 33.1 ; 30.8 ; 30.5$; $26.8 ; 23.7 ; 22.8 ; 14.4$. ESI MS: $m / z 344[\mathrm{M}+\mathrm{H}]+$. Anal Calcd. for $\mathrm{C}_{20} \mathrm{H}_{41} \mathrm{NO}_{3}: \mathrm{C}, 69.92 ; \mathrm{H}, 12.03 \%$. Found: $\mathrm{C}$, 70.04; H, $12.00 \%$.

\subsection{1,2-Diacetyl D-erythro-sphinganine (4)}

To a solution of 1,3-diol $\mathbf{1 3}(0.1 \mathrm{~g}, 0.29 \mathrm{mmol})$ in dry $\mathrm{CH}_{2} \mathrm{Cl}_{2}(4 \mathrm{~mL}), \mathrm{Et}_{3} \mathrm{~N}(0.04 \mathrm{~mL}, 0.29 \mathrm{mmol})$ was added, followed by $\mathrm{Ac}_{2} \mathrm{O}(0.027 \mathrm{~mL}, 0.29 \mathrm{mmol})$ was added at $0^{\circ} \mathrm{C}$ and the reaction mixture was allowed to rise to r.t. After completion of reaction $(2 \mathrm{~h})$, the reaction mixture was washed with brine $(5 \mathrm{~mL})$ and water $(5 \mathrm{~mL})$, dried over $\mathrm{Na}_{2} \mathrm{SO}_{4}$ and evaporated. The crude product was purified by silica gel CC using (AcOEt/hexane,
2:8) to give pure 1,2-diacetyl D-erythro-sphinganine $\mathbf{4}$ $(0.088 \mathrm{~g}, 79 \%)$ as a white solid. $[\alpha]_{\mathrm{D}}^{25}=+6.2(c=0.25$, $\left.\mathrm{CH}_{3} \mathrm{OH}\right)$. IR $\tilde{\mathrm{o}}_{\max }(\mathrm{KBr}) / \mathrm{cm}^{-1}: 3279,2918,2850,1739$, 1650, 1555, 1465, 1341, 1221. ${ }^{1} \mathrm{H}$ NMR $(500 \mathrm{MHz}$, $\left.\mathrm{CDCl}_{3}\right): 5.98(d, J=7.9 \mathrm{~Hz}, 1 \mathrm{H}) ; 4.33(d d, J=11.6$, $6.4 \mathrm{~Hz}, 1 \mathrm{H}) ; 4.18(d d, J=11.6,3.2 \mathrm{~Hz}, 1 \mathrm{H}) ; 4.11$ $(m, 1 \mathrm{H}) ; 3.63(m, 1 \mathrm{H}) ; 2.06(s, 3 \mathrm{H}) ; 2.00(s, 3 \mathrm{H}) ; 1.47$ $(m, 2 \mathrm{H}) ; 1.24$ (br. $s, 26 \mathrm{H}) ; 0.86(t, J=6.8 \mathrm{~Hz}, 3 \mathrm{H})$. ${ }^{13} \mathrm{C}$ NMR $\left(125 \mathrm{MHz}, \mathrm{CDCl}_{3}\right): 171.3 ; 170.3 ; 72.6 ; 63.1$; $52.9 ; 34.0 ; 31.9 ; 29.6 ; 29.6 ; 29.5 ; 29.3 ; 25.9 ; 23.3 ; 22.7$; 20.9; 14.1. ESI MS: $m / z 386[\mathrm{M}+\mathrm{H}]^{+}$. Anal Calcd. for $\mathrm{C}_{22} \mathrm{H}_{43} \mathrm{NO}_{4}$ : C, 68.53; H, 11.24\%. Found: C, 68.70; H, $11.21 \%$.

\section{Results and Discussion}

The retrosynthetic analysis (Scheme 1) indicates that the 1,2-diacetyl D-erythro-sphinganine $\mathbf{4}$ can be synthesized from the D-erythro-sphinganine triacetate $\mathbf{6}$, which can be prepared from the monoacetyl ester 8 . On the other hand, L-threo-sphinganine triacetate 7 can be prepared from its diacetyl compound $\mathbf{5}$, which can also be prepared from the same monoacetyl ester $\mathbf{8}$. The monoacetyl compound $\mathbf{8}$ can in turn, be prepared from the Garner aldehyde 9.

The present synthesis was initiated by treatment of the Garner aldehyde 9 with 1-bromo pentadecane and $\mathrm{Mg}$ in $\mathrm{THF}$ at $-78^{\circ} \mathrm{C}$ and the mixture was brought to r.t. to produce the diastereomeric alcohols 10 and 10a (Scheme 2). The stereochemistry of the two diastereomers 10 and 10a were assigned by following Buono's method. ${ }^{9}$ According to this method, syn alcohol is major product compared to anti-alcohol. This stereochemistry is further confirmed by subsequent conversion to the target molecules. The two diastereomers were separated by careful CC (silica gel 100-200 mesh, 0-5\% increasing amount of AcOEt in hexane), which produced $\mathbf{1 0}$ and 10a in the ratio 9:1 (syn:anti, 9:1) with $86 \%$ yield.

Due to less amount of minor diastereomer 10a, we have synthesized 1,2-diacetyl D-erythro-sphinganine (4) and D-erythro-sphinganine triacetate (6) from major diastereomer $\mathbf{1 0 .}$

The acetonide group of alcohol $\mathbf{1 0}$ was deprotected by treatment with PPTS in $\mathrm{MeOH}$ to form diol 11 with $92 \%$ yield $^{10}$ (Scheme 3). The 1,3-diol 11 was mono protected of its primary hydroxyl group as acetyl ester 8 by treatment with $\mathrm{Ac}_{2} \mathrm{O}$ and $\mathrm{Et}_{3} \mathrm{~N}$ in $\mathrm{CH}_{2} \mathrm{Cl}_{2}$ with $77 \%$ yield. ${ }^{11}$ The mono acetyl ester $\mathbf{8}$ was converted to 1,2-diacetyl L-threo-sphinganine $\mathbf{5}$ by following two steps: i) Boc group was deprotected from 8 by using excess TFA in $\mathrm{CH}_{2} \mathrm{Cl}_{2}$; ii) unprotected amine was converted to $\mathrm{N}$-acylated amine on treatment with acetyl chloride, aq. $\mathrm{NaHCO}_{3}$ in $\mathrm{CH}_{2} \mathrm{Cl}_{2}$ with $72 \%$ yield. ${ }^{12}$ 


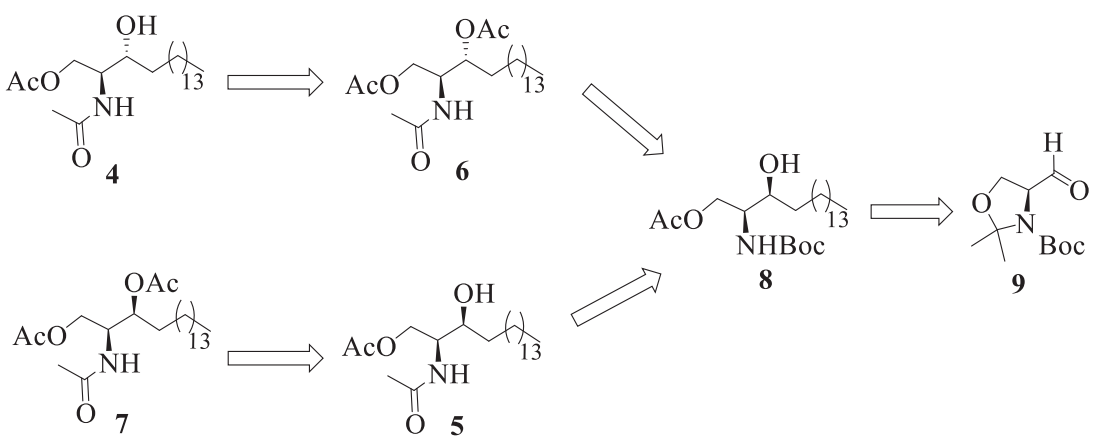

Scheme 1. Retrosynthetic analysis of sphingolipids.

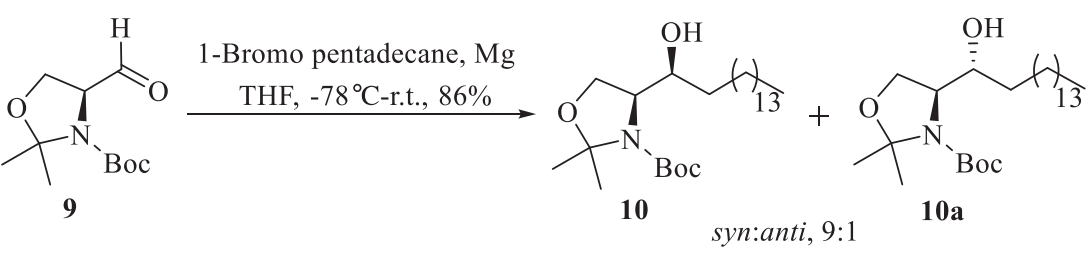

Scheme 2. Grignard reaction on Garner aldehyde.

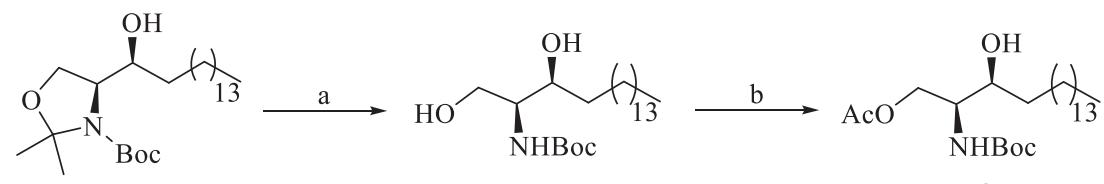

10

11

8

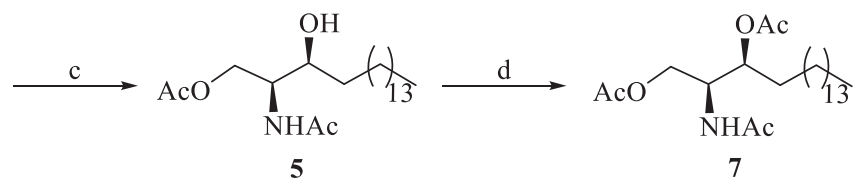

Scheme 3. (a) PPTS/MeOH, r.t., 2 h, $92 \%$. (b) $\mathrm{Ac}_{2} \mathrm{O}, \mathrm{Et}_{3} \mathrm{~N}, \mathrm{CH}_{2} \mathrm{Cl}_{2}, 0{ }^{\circ} \mathrm{C}$-r.t., 2 h, $77 \%$. (c) i) TFA $/ \mathrm{CH}_{2} \mathrm{Cl}_{2}$, r.t., 1 h. ii) Acetyl chloride, $\mathrm{NaHCO}_{3}, \mathrm{CH}_{2} \mathrm{Cl}_{2}$ $\mathrm{H}_{2} \mathrm{O}$, r.t., $2.5 \mathrm{~h}, 72 \%$ (Over two steps). (d) $\mathrm{Ac}_{2} \mathrm{O}, \mathrm{Et}_{3} \mathrm{~N}, \mathrm{CH}_{2} \mathrm{Cl}_{2}, 0{ }^{\circ} \mathrm{C}$-r.t., $2 \mathrm{~h}$, $79 \%$.

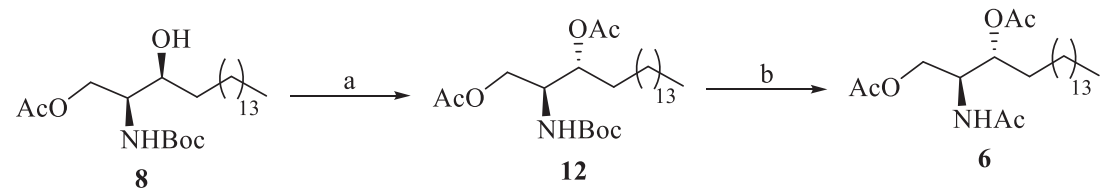

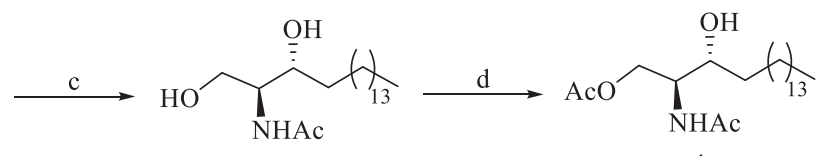

13

Scheme 4. (a) $\mathrm{PPh}_{3}, \mathrm{AcOH}, \mathrm{DIAD}$, THF, $0{ }^{\circ} \mathrm{C}$-r.t., 4 h, 77\%. (b) i) TFA/ $\mathrm{CH}_{2} \mathrm{Cl}_{2}$, r.t., 1 h. ii) $\mathrm{AcCl}, \mathrm{NaHCO}_{3}, \mathrm{CH}_{2} \mathrm{Cl}_{2}-\mathrm{H}_{2} \mathrm{O}$, r.t., 2.5 h, $73 \%$ (Over two steps). (c) $\mathrm{Na}_{2} \mathrm{CO}_{3} / \mathrm{MeOH}$, r.t., 1 h, $82 \%$. (d) $\mathrm{Ac}_{2} \mathrm{O}, \mathrm{Et}_{3} \mathrm{~N}, \mathrm{CH}_{2} \mathrm{Cl}_{2}, 0{ }^{\circ} \mathrm{C}$-r.t., $2 \mathrm{~h}, 79 \%$.

1,2-Diacetyl L-threo-sphinganine 5 was converted to Lthreo-sphinganine triacetate 7 by treatment with $\mathrm{Ac}_{2} \mathrm{O}$ and $\mathrm{Et}_{3} \mathrm{~N}$ in $\mathrm{CH}_{2} \mathrm{Cl}_{2}$ with $79 \%$ yield.
The mono acetyl ester $\mathbf{8}$ was subjected to Mitsunobu inversion by using triphenyl phosphine $\left(\mathrm{PPh}_{3}\right), \mathrm{AcOH}$ and diisopropyl azodicarboxylate (DIAD) in dry THF 
to obtain 1,3-diacetyl compound $\mathbf{1 2}$ with $77 \%$ yield $^{13}$ (Scheme 4). 1,3-Diacetyl compound $\mathbf{1 2}$ was converted to D-erythro-sphinganine triacetate $\mathbf{6}$ by following two steps: i) Boc group was deprotected from $\mathbf{1 2}$ by using excess TFA in $\mathrm{CH}_{2} \mathrm{Cl}_{2}$; ii) unprotected amine was converted to $\mathrm{N}$-acylated amine on treatment with $\mathrm{AcCl}$, aq. $\mathrm{NaHCO}_{3}$ in $\mathrm{CH}_{2} \mathrm{Cl}_{2}$ with $73 \%$ yield. D-erythrosphinganine triacetate $\mathbf{6}$ was converted to 1,3-diol $\mathbf{1 3}$ by treatment with $\mathrm{Na}_{2} \mathrm{CO}_{3}$ in $\mathrm{MeOH}^{14}$ at r.t. with $82 \%$ yield. The 1,3-diol 13 was mono protected of its primary hydroxyl group as acetyl ester by treatment with $\mathrm{Ac}_{2} \mathrm{O}$ and $\mathrm{Et}_{3} \mathrm{~N}$ in $\mathrm{CH}_{2} \mathrm{Cl}_{2}$ to produce 1,2-diacetyl Derythro-sphinganine 4 with $79 \%$ yield. The physical (optical rotation) and the spectral $\left({ }^{1} \mathrm{H}\right.$ and ${ }^{13} \mathrm{C}$ NMR and MS) properties of $\mathbf{4}$ were found to be identical to those reported for the naturally occurring compound. ${ }^{6}$

\section{Conclusions}

In conclusion, we have described the stereoselective total synthesis of naturally occurring sphingolipid 1,2-diacetyl D-erythro-sphinganine (4) along with three other sphingolipids, namely, 1,2-diacetyl Lthreo-sphinganine (5) (C-3 epimer of 4), D-erythrosphinganine triacetate (6) (triacetyl derivative of compound 2) and L-threo-sphinganine triacetate (7) (triacetyl derivative of compound 3, Safingol). Synthesis of 1,2-Diacetyl D-erythro-sphinganine (4) and 1, 2-diacetyl L-threo-sphinganine (5) are reported here for the first time.

\section{Supplementary information (SI)}

All the copies of ${ }^{1} \mathrm{H}$ NMR and ${ }^{13} \mathrm{C}$ NMR are given in the supporting information. Supplementary Information is available at www.ias.ac.in/chemsci.

\section{Acknowledgments}

The authors thank CSIR and UGC, New Delhi for grant of fellowships and financial assistance.

\section{References}

1. Hakomori S 1990 J. Biol. Chem. 26518713

2. Van Meer G and Burger K N J 1992 Trends Cell Biol. 2332

3. Mer J N and Hakomori S 1983 In Hand book of Lipid Research Vol. 3, Sphingolipid Biochemistry D J Hanahan (Ed.) (New York: Plenum Press)

4. USP Dictionary of USAN and International Drug Names (US Pharmacopeia: Rockville, MD, 2000636)

5. Schwartz G K, Jiang J, Kelsen D and Albino A P 1993 J. Natl. Cancer Inst. $\mathbf{8 5} 402$

6. Choi J-H, Yoshida M, Suzuki T, Harada E, Kawade M, Yazawa K, Nishimoto S, Hirai H and Kawagishi H 2013 Tetrahedron 698609

7. (a) Paramesh J, Kumar C G, Poornachandra Y and Das B 2015 Synthesis 47 653; (b) Bhunia N and Das B 2015 Synthesis 47 1499; (c) Lingaiah M, Kumar C G, Poornachandra Y and Das B 2015 Tetrahedron Lett. 56 4631; (d) Reddy P R and Das B 2015 Helv. Chim. Acta 98 509; (e) Srilatha M and Das B 2015 Helv. Chim. Acta 98 267; (f) Reddy N S and Das B 2015 Helv. Chim. Acta 9878

8. (a) Shibuya H, Kawashima K, Ikeda M and Kitagawa I 1989 Tetrahedron Lett. 30 7205; (b) Cook G R and Pararajasingham K 2002 Tetrahedron Lett. 43 9027; (c) Mori K and Umemura T 1981 Tetrahedron Lett. 22 4433; (d) Umemura T and Mori K 1987 Agric. Biol. Chem. 51 1973; (e) Ravinder M, Narendar T R, Sunday O O, Ramesh V and Rao V J 2012 Arkivoc vi 421; (f) Azuma H, Tamagaki S and Ogino K $2000 \mathrm{~J}$. Org. Chem. 65 3538; (g) Ndakala A J, Hashemzadeh M, So R C and Howell A R 2002 Org. Lett. 41719

9. Villard R, Fotiadu F and Buono G 1998 Tetrahedron: Asymmetry 9607

10. Lin S, Yang Z-Q, Kwok B H B, Koldobskiy M, Crews C M and Danishefsky S J $2004 \mathrm{~J}$. Am. Chem. Soc. 1266347

11. Yadav J S, Aravind S, Kumar G M and Reddy B V S 2012 Tetrahedron Lett. 536163

12. Mina J G, Mosely J A, Ali H Z, Denny P W and Steel P G 2011 Org. Biomol. Chem. 91823

13. Wallner A, Mang H, Glueck S M, Steinreiber A, Mayer S F and Faber K 2003 Tetrahedron: Asymmetry 14 2427

14. Devijver C, Salmoun M, Daloze D, Braekman J C, De Weerdt W H, De Kluijver M J and Gomez R 2000 J. Nat. Prod. 63978 